\title{
Clustering-led complex brain networks approach
}

\author{
Dazhong Liu ${ }^{\mathrm{a},{ }^{*}}$ and Ning Zhong ${ }^{\mathrm{b}}$ \\ ${ }^{a}$ School of Mathematics and Computer Science, Hebei University, Baoding 071002, China \\ ${ }^{b}$ Department of Life Science and Informatics, Maebashi Institute of Technology, Maebashi 371-0816, \\ Japan
}

\begin{abstract}
This paper reviewed the meaning of the statistic index and the properties of the complex network models and their physiological explanation. By analyzing existing problems and construction strategies, this paper attempted to construct complex brain networks from a different point of view: that of clustering first and constructing the brain network second. A clustering-guided (or led) construction strategy towards complex brain networks was proposed. The research focused on the discussion of the task-induced brain network. To discover different networks in a single run, a combined-clusters method was applied. Afterwards, a complex local brain network was formed with a complex network method on voxels. In a real test dataset, it was found that the network had small-world characteristics and had no significant scale-free properties. Meanwhile, some key bridge nodes and their characteristics were identified in the local network by calculating the betweenness centrality.
\end{abstract}

Keywords: Complex brain network, functional connectivity, clustering, functional magnetic resonance imaging

\section{Introduction}

The human brain is considered to be one of the real world's most complex systems. Understanding how to construct a network is a fundamental part of complex network analysis. Furthermore, the principles of brain organization are functional separation and integration, and both have features of complex networks [1]. In order to further research on the overall characteristics of brain function, one needs some other theoretical model, namely the complex network theory. The complex network exists in various fields of science, such as biology, physics, information science and social science. With the data obtained from neuroimaging, one can construct a complex network based upon graph theory, analyze its topology and dynamics principle, and then understand the working mechanism and characters inside the brain. Because of technical limitations, human brain networks are primarily built at a large-scale, based on voxel level or on a priori anatomical template of the brain region.

In addition, more achievements have been made on brain functional network formation than on brain structural network construction. For example, Salvador and his colleagues [2] first created a brain functional network using resting state data. Functional magnetic resonance imaging (fMRI)

\footnotetext{
${ }^{*}$ Corresponding author: Dazhong Liu, School of Mathematics and Computer Science, Hebei University, Baoding 071002, China. Tel.: 86-0312-5079351; Fax: 86-0312-5016914; E-mail: liudazhong@hbu.edu.cn.
} 
images were divided into 90 regions according to a priori anatomical template. Then the coefficients of partial correlation between these areas were computed, and the existence of connection was confirmed by counting. Finally, a functional network was obtained. By network analyzing, the smallworld properties of the brain network were determined. In the same year, Eguíluz [3] first built an entire brain functional network with data acquired when the brain was on task. He also found that brain functional networks that were based on voxel level also had small-world properties, and he confirmed that the brain functional network nodes exhibited scale-free power-law distributions. Moreover, when constructing brain networks, one main method is to select some brain areas as the seed of a region of interest (ROI) in advance, and then compute its correlation between other areas. Another method is to evaluate the correlation of all brain voxels.

Different methods were proposed in this paper. First, the relevant local brain network, like the default network or task-related activity network, was determined by the proposed clustering method. Then, the complex network characteristics and the relationship between each local network were analyzed. From which, a more detailed understanding of the brain function characters of local networks was obtained. Hence, the ignorance of some aspects caused by the above overall calculation method was overcome.

In this article, a new clustering-guided construction approach towards complex brain networks is provided. We begin by a non-technical introduction to the important conceptual issues associated with complex networks' statistical properties, and then, the proposed method is described, and a detailed case study is presented. Finally, some of the issues associated with traditional methods are discussed, and concluding remarks are given.

\section{Statistical properties of complex network}

When studying a complex network, four types of topology are considered: regular network, random graph, small-world network and scale-free network. A small-world network is a type of network with a relatively shorter average path length and a relatively higher clustering coefficient. To quantitatively judge a network's small-world property, one needs to use a random network as reference and then compare them at the same scale. If the network tested has a relatively higher clustering coefficient and approximately the shortest path length in comparison with the random network (that is to say, $\left.\gamma=\mathrm{C} / \mathrm{C}_{\text {rand }}>>1, \lambda=\mathrm{L} / \mathrm{L}_{\text {rand }} \sim 1\right)$, then one can say that this network is a small-world network. In the aforementioned equation, $\mathrm{C}_{\text {rand }}$ stands for the clustering coefficient of a random network, $\mathrm{C}$ stands for the clustering coefficient of the network being tested, L stands for the average length of the network being tested, and $\mathrm{L}_{\text {rand }}$ stands for the average path length of a random network. One can also combine the two indexes into a scalar $s=\gamma / \lambda$ to measure the small-worldness; for example, when $s>1$, the network has small-world property, and the larger $\mathrm{s}$ is, the more intense its small-world property [4-8].

The degree distribution of both a random network and a regular network presents a sort of homogeneous network. A scale-free network like the World Wide Web is different. Its node degree distribution follows power-law distribution, $\mathrm{P}(\mathrm{k}) \sim \mathrm{k}^{-\alpha}$, where $\mathrm{k}$ stands for the degree of nodes, and $\alpha$ is an exponent (which is usually assigned a value from 2 to 4). In double logarithmic coordinates, the plot of this kind of distribution is similar to a line. In a scale-free network, a few nodes have many connections to other nodes, and their degrees are very high; however, most nodes only have a few connections, with only tiny degrees. The scale-free network turns out to be robust when facing random attack, but it is fragile and sensitive when confronting the attempted attack [4-8]. 


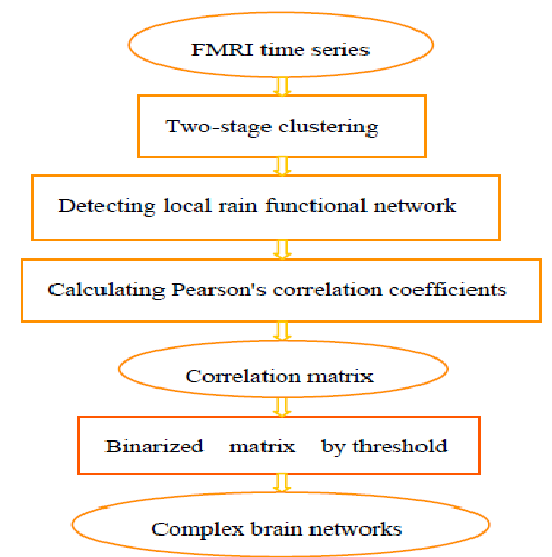

Fig. 1. Flowchart of the construction of complex brain network.

\section{Complex network analysis method for fMRI data}

Neuroscientific investigations have shown that different experimental tasks will activate different brain regions. Each brain region is composed of a number of voxels, and each voxel has its own time series. According to the concept of brain functional connectivity, brain regions that are not adjacent spatially can be correlated when neural physiological events occur. These correlated regions then form the brain functional network. Experimental tasks will activate a different brain functional network, which can be regarded as a different local brain functional network. However, the complex network features for each local brain functional network still need to be studied further. The proposed method is as follows: the local brain networks were first determined according to the local brain network detection method. Then, within each local brain network, each voxel was regarded as a node. The connections between nodes were set up with the method proposed by Eguíluz [3]. Thus, a local complex network was formed. Finally, the properties of the local complex brain network were analyzed. The analysis method was divided into three steps; referring to Figure 1 will provide an outline of how these steps were conducted [9]. Here are the introductions to the process:

\subsection{Local brain functional network detection}

After pre-processing the fMRI data, the two-stage clustering method (TSCA), which was suggested in [9], was used to process data and obtain a number of brain functional networks. The ith brain network is denoted as Net . $_{\text {. }}$.

TSCA is constituted of two clustering methods, K-means and Affinity Propagation (AP) [10]. For each clustering algorithm, a specific similarity measure was adopted. The Euclidean distance of autocovariance value series (AVS) of each pair of voxels was used as a similarity measure by Kmeans. Firstly, K-means clustered datasets into groups. Then AP method selected a particular group for further clustering. The benefits of the two-stage approach were: first, the datasets are very large, and if AP is used individually, the similarity matrix will run out of all the memory; second, if we use $\mathrm{K}$-means solely, we are not able to identify the appropriate clustering number. The AVS of voxel $\mathrm{k}$ is calculated with the Eq. (1). 


$$
A V S_{\tau}(k)=E\left\{\left(y_{n+\tau}(k)-m(k)\right)\left(y_{n}(k)-m(k)\right)\right\}
$$

where $\left\{y_{n}(k)\right\}$ are the fMRI signals of the dataset, $n$ denotes the time indexes, $m(k)$ represents the average value of the signals of voxel $k$, and $\tau$ is known as the delay.

\subsection{Construction of local complex brain network}

After establishing the voxels of a local brain network, a connection is set up using Eguíluz's method. The Pearson's correlation coefficients (cps) from all of the voxels in every $\mathrm{Net}_{\mathrm{i}}$ are calculated, and then, the cps constitute the correlation matrix.

$$
c p=\frac{\sum\left(r_{i}-\bar{r}_{i}\right)\left(r_{j}-\bar{r}_{j}\right)}{\sqrt{\sum\left(r_{i}-\bar{r}_{i}\right)^{2}} \sqrt{\sum\left(r_{j}-\bar{r}_{j}\right)^{2}}}
$$

where $r_{i}$ and $r_{j}$ are the time series of voxel $\mathrm{i}$ and $\mathrm{j}$ with means of $\bar{r}_{i}$ and $\overline{r_{j}}$, respectively. Given a threshold value $r$, according to the correlation coefficient of two voxels' time series, it is determined whether there is a connection between the nodes in the network. If the correlation coefficient is bigger than $r$, there is an edge connecting the two voxels. With this method, a binarized matrix can be obtained by a different threshold.

\subsection{Analysis of complex network properties}

For the local complex network composed, the following is caluclated: the clustering coefficient, betweenness centrality, average path length and degree distribution. Furthermore, its small-world property and scale-free property are analyzed, and its pivotal nodes are found.

\section{Case study for constructing complex network}

To elucidate the aforementioned method on a representative dataset, a set of auditory data was chosen from the Wellcome Department of Imaging Neuroscience of the University College London (http://www.fil.ion.ucl.ac.uk/spm/data/). Participants were scanned on a 2.0 Tesla Siemens MRI scanner with the following parameters: repetition time $=7 \mathrm{~s}$, matrix $=64 \times 64 \times 64$, and voxel size $=3 \mathrm{~mm} \times 3 \mathrm{~mm} \times 3 \mathrm{~mm}$. FMRI data was obtained in one session using gradient echo planar imaging. T1 anatomical scans were also collected with voxel size $=1 \mathrm{mmx} 1 \mathrm{mmx} 1 \mathrm{~mm}$. The first twelve functional volumes were discarded to eliminate $\mathrm{T} 1$ effects. The other 84 functional volumes were preprocessed to analyze. The paradigm consisted of eight repeated periods of rest and auditory task, starting with a rest. During the task trials, bi-syllabic words were presented binaurally at a rate of 60 per minute.

By the first step, three local brain functional networks were obtained, namely the default mode network (DMN, in blue), auditory task-induced network (in orange), and dorsal attention network (in green), as shown in Figure 2. The map is rendered by using xjView (http://www.alivelearn.net/xjview/). For the next step, a complex network for each local network was 


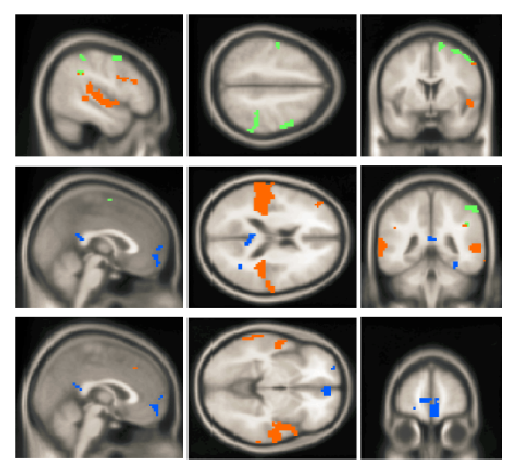

Fig. 2. The brain overlay maps of TSCA results. Blue: default mode network, orange: auditory task-induced network, and green: dorsal attention network.

constructed. In constructing a binary network for the three local brain networks, the threshold value $\mathrm{r}$ was successively set to be $0.7,0.8$ and 0.9 . After calculation, the corresponding numerical chart and analysis result are given. Experimental results showed that the voxels contained in each brain network were time-dependent as well as exhibited a high degree of coherence. According to the aforementioned methods, we constructed a graphic network adjacency matrix and calculated the average path length, node degree, degree distribution, betweenness centrality, clustering coefficient, and network small-worldness $S=\gamma / \lambda$, which are shown in Table 1 [9]. The calculations were aided by NodeXL (http://nodexl.codeplex.com/).

For all three $r$ values, the network small-worldness of the default network, attention network and auditory network were all greater than 1 , meaning these three networks all had small-world properties and were robust to parameter $r$. This result is consistent with the result mentioned in the introduction, but here the analysis results are all about the local brain network based on voxels.

For the scale-free properties, the threshold value was successively set to be $0.7,0.8$ and 0.85 . It was inspected whether the degree distribution or cumulative distribution approximated a straight line on a double logarithmic plot. This method may give rise to a biased conclusion [11]. By using the fitting test method (given in [11]) and synthetic power-law data by the Monte Carlo method and then by using the Kolmogorov-Smirnov statistic to compute the p-value, one can draw the conclusion that the data might not obey power-law distribution if the p-value is significantly less than 1 [12].

The degree distribution figure and the cumulative distribution figure of the auditory task-induced network (A-net), with a threshold value of 0.85, are shown in Figure 3; however, figures illustrating the default mode network and dorsal attention network are omitted here due to space limitations. In the cumulative distribution graph, the fitted curve to calculate the power-law exponent is given; Table 2 gives the correlated value. The node number did not include zero degree nodes, and the power-law exponent and the power-law standard deviations were the estimated values from the fitted curve. From Table 2, it can be noted that all the p-values were less than 0.05 and did not meet the power-law distribution. Furthermore, it was not rigorous to directly inspect whether the degree distribution graph approximates a straight line on a double logarithmic plot.

Concerning whether the brain network satisfies power-law distribution, there are mixed experimental results. However, some literatures have reported that a brain functional network satisfied exponentially truncated power-law rather than scale-free power-law distribution, and it tended to decay rapidly at the tail of the graph [13-15]. These reports are similar to the experimental results obtained from this paper. 
Table 1

Small-world properties of the three brain functional networks

\begin{tabular}{|l|l|l|l|l|l|l|}
\hline & $\begin{array}{l}\text { threshold } \\
\text { value }\end{array}$ & $\begin{array}{l}\text { average path } \begin{array}{l}\text { length } \\
\text { (random) }\end{array} \\
\text { average path }\end{array}$ & $\begin{array}{l}\text { clustering } \\
\text { length (tested) } \\
\text { (random) }\end{array}$ & $\begin{array}{l}\text { clustering } \\
\text { coefficient } \\
\text { (tested) }\end{array}$ & $\mathrm{S}=\gamma / \lambda$ \\
\hline \multirow{2}{*}{$\begin{array}{l}\text { Default } \\
\text { network }\end{array}$} & 0.7 & 2.30 & 3.16 & 0.23 & 0.71 & 2.25 \\
\cline { 2 - 7 } & 0.8 & 2.81 & 2.81 & 0.10 & 0.59 & 6.02 \\
\cline { 2 - 7 } & 0.9 & 4.12 & 2.74 & 0.02 & 0.25 & 18.84 \\
\hline \multirow{2}{*}{$\begin{array}{l}\text { Attention } \\
\text { Network }\end{array}$} & 0.7 & 1.86 & 2.00 & 0.39 & 0.67 & 1.60 \\
\cline { 2 - 7 } & 0.8 & 2.38 & 3.28 & 0.12 & 0.65 & 3.93 \\
\hline \multirow{2}{*}{$\begin{array}{l}\text { Auditory } \\
\text { Network }\end{array}$} & 0.9 & 3.48 & 5.12 & 0.04 & 0.49 & 9.30 \\
\cline { 2 - 7 } & 0.8 & 1.76 & 1.88 & 0.53 & 0.69 & 1.24 \\
\cline { 2 - 7 } & 0.9 & 2.10 & 3.09 & 0.36 & 0.63 & 1.19 \\
\hline
\end{tabular}

The bigger a node's betweenness centrality, the more important role it plays in information transmission and organization inside the network. The top 7 nodes of betweenness centrality for DMN add up to account for $33.5 \%$ of the summation in this network. Similarly, the top 5 nodes of betweenness centrality for the dorsal attention network add up to account for $33.7 \%$ of the summation in this network. The top 8 nodes of betweenness centrality for the auditory active network add up to account for $16.6 \%$ of the summation the betweenness centrality in this network. Interestingly, in the auditory active network, the points with the highest betweenness centrality are all located on the right superior temporal gyrus. This may reflect the lateralized interpretation of auditory cortex in some task stimulation [16].

\section{Concluding remarks}

By analyzing the three local brain functional networks, it was discovered that all of them had smallworld properties. We also analyzed their degree distribution properties.

This method was based on voxels level, quite different from the 'seed' choosing method (based on $a$ priori anatomical knowledge). The strategy for building a brain network was different from the existing whole-brain strategy. The local brain functional network region was initially taken into consideration, and a better understanding was obtained concerning the relationship between whole and local brain networks.

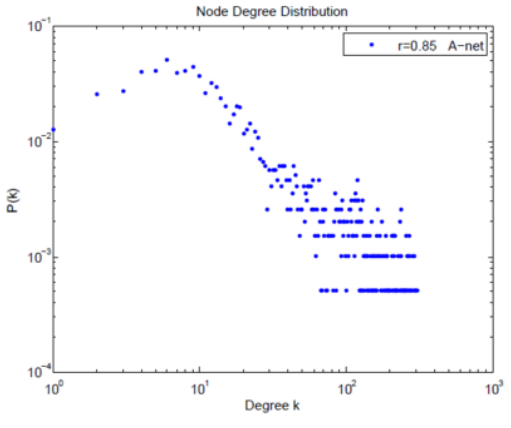

(a)

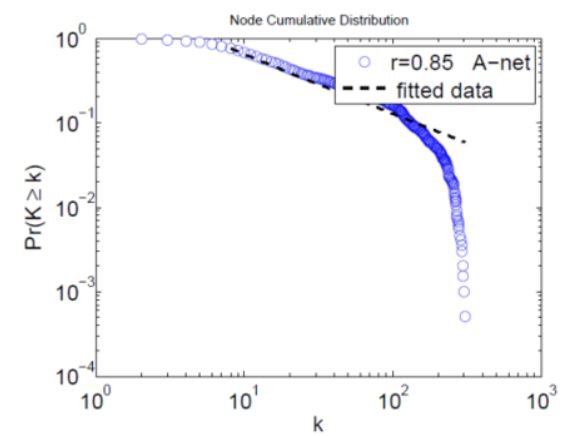

(b)

Fig. 3. (a) Degree distribution for auditory active network; (b) Degree cumulative distribution for auditory active network $(\mathrm{r}=0.85)$ 
Table 2

Scale-free properties of the three brain functional networks

\begin{tabular}{|c|c|c|c|c|c|}
\hline & threshold value & Exponent $\alpha$ & \begin{tabular}{|l|} 
standard \\
deviations \\
\end{tabular} & p-value & $\begin{array}{|ll|}\begin{array}{l}\text { number } \\
\text { nodes }\end{array} & \text { of } \\
\end{array}$ \\
\hline \multirow{3}{*}{$\begin{array}{l}\text { Default } \\
\text { network }\end{array}$} & 0.7 & 2.4383 & 0.2033 & 0.00 & 190 \\
\hline & 0.8 & 2.7069 & 0.5659 & 0.04 & 179 \\
\hline & 0.85 & 2.6576 & 0.3601 & 0.01 & 170 \\
\hline \multirow{3}{*}{$\begin{array}{l}\text { Attention } \\
\text { Network }\end{array}$} & 0.7 & 3.446 & 0.1546 & 0.00 & 242 \\
\hline & 0.8 & 3.4416 & 0.2388 & 0.00 & 234 \\
\hline & 0.85 & 3.1500 & 0.3622 & 0.00 & 227 \\
\hline \multirow{3}{*}{$\begin{array}{l}\text { Auditory } \\
\text { Network }\end{array}$} & 0.7 & 3.5 & 0.000 & 0.00 & 1988 \\
\hline & 0.8 & 2.6215 & 0.5992 & 0.00 & 1980 \\
\hline & 0.85 & 1.6916 & 0.0236 & 0.00 & 1974 \\
\hline
\end{tabular}

After we had determined a brain functional network with assistance from TSCA, we chose a relatively higher threshold value $(r>0.6)$ when constructing a binary network, primarily because of the high correlation coefficient in each network. Interestingly, though every network had a physical meaning, it displayed 'small-world' properties locally. This characteristic was robust to the threshold value $r$. The results from this analysis showed that degree distribution did not follow power-law distribution, and the distribution decayed exponentially at the tail of the graph. A key feature of this kind of distribution is that the probability for the occurrence of points in a higher degree is smaller than that of power-law distribution. This feature is of very practical significance. In practice, nodes with a very high degree can be isolated so that they do not have a connection with faraway nodes with a high degree.

If one constructs a complex network with the method given by article [3], it will either lead to confusion or neglect problems because the process chooses a certain area and then conducts correlation tests with whole brain voxels. For example, if one chooses some auditory activated region, then voxels of the attention network may be recruited because the time series of the first several periods are similar to each other. Thus, in this method, the correlation coefficient will be high and will cause confusion. On the other hand, if one selects only positive activation, one may ignore the negative activation network. As a result, the approach could alleviate the problem by clustering first. This strategy was different from the commonly used strategy of constructing a complex brain network. It was based on the data-driven brain analysis method.

This study focused on the construction of complex networks based on functional connectivity; however, it did not consider effective connectivity. Hence, calculation of the correlation coefficient is a common practice. Concerning this, literature [17] pointed out that the partial correlation is more related to effective connectivity analysis, and literature [18] chose multivariate mutual information as the measure to estimate functional connectivity. Both methods are based on specified ROIs. However, computing complicity needs to be considered for the voxel-wise situation in the study.

We used partial correlation to examine the relationship among the three networks. The partial correlation coefficients (with $\mathrm{p}<0.005$ ) between DMN and A-net, DMN and dorsal attention network, and A-net and dorsal attention network were $-0.441,0.322$ and 0.879 , respectively, which reflects the holistic correlation between any two networks conditioned on the remaining network.

Despite having some advantages shown in the present study, the method is still in the developing stage with much more yet to be done. The experimental dataset is simple, so much more complex 
brain networks are not concerned. Some other computing measurements need to be considered, such as mutual information and partial correlation [19]. Further systematic brain research [20] is therefore warranted.

\section{Acknowledgment}

The work is supported in part by the National Natural Science Foundation of China under Grant (No. 61170039, No. 61375075), the Natural Science Foundation of Hebei Province (No. F2012201006, No. F2012201020) and the NSF of Hebei University (2013-251).

\section{References}

[1] K.J. Friston, Functional and effective connectivity in neuroimaging: a synthesis, Human Brain Mapping 2 (1994), 5678.

[2] R. Salvador, J. Suckling, M.R. Coleman et al., Neurophysiological architecture of functional magnetic resonance images of human brain, Cerebral Cortex 15 (2005), 1332-1342.

[3] V.M. Eguíluz, D.R. Chialvo, G.A. Cecchi, M. Baliki and A.V. Apkarian, Scale-free brain functional networks, Physical Review Letters 94 (2005), 1-4.

[4] M. Rubinov and O. Sporns, Complex network measures of brain connectivity: Uses and interpretations, NeuroImage 52 (2010), 1059-1069.

[5] J.C. Reijneveld, S.C. Ponten, H.W. Berendse and C.J. Stam, The application of graph theoretical analysis to complex networks in the brain, Clin. Neurophysiol. 118 (2007), 2317-2331.

[6] P. Fransson, Spontaneous low-frequency BOLD signal fluctuations: An fMRI investigation of the resting-state default mode of brain function hypothesis, Human Brain Mapping 29 (2005), 15-29.

[7] D.J. Watts and S.H. Strogatz, Collective dynamics of "small-world" networks, Nature 393 (1998), 440-442.

[8] A.L. Barabasi and R. Albert, Emergence of scaling in random networks, Science 286 (1999), 509-512.

[9] D. Liu, Clustering based analysis for human brain functional magnetic resonance imaging dataset, Ph.D. Dissertation, Beijing University of Technology, 2012.

[10] B. J. Frey and D. Dueck, Clustering by passing messages between data points, Science 315 (2007), 972-976.

[11] A. Clauset, C.R. Shalizi and M.E.J. Newman, Power-law distributions in empirical data, SIAM Review 51 (2009), 661703.

[12] Lev Muchnik, Royi Itzhack, Sorin Solomon and Yoram Louzoun, Self-emergence of knowledge trees: Extraction of the Wikipedia hierarchies, Phys. Rev. E 76 (2007), 016106-1-016106-12.

[13] S. Achard, R. Salvador, B. Whitcher, J. Suckling and E. Bullmore, A resilient, low frequency, small-world human brain functional network with highly connected association cortical hubs, J. Neurosci. 26 (2006), 63-72.

[14] E. Bullmore and O. Sporns, Complex brain networks: graph theoretical analysis of structural and functional systems, Nat. Rev. Neurosci. 10 (2009), 186-198.

[15] D. Liu, N. Zhong and Y. Qin, Exploring functional connectivity networks in fMRI data using clustering analysis, Brain Informatics-International Conference LNAI6889 (2011), 148-159.

[16] H.S. Kim, M.H. Choi, H.J. Yoon, H.J. Kim, U.H. Jeoung, S.J. Park, D.W. Lim, S.C. Chung and B.-Y. Lee, Cerebral activation and lateralization due to the cognition of a various driving speed difference: An fMRI study, Bio-Medical Materials and Engineering 24 (2014), 1133-1139.

[17] G. Marrelec, A. Krainik, H. Duffau, M. Pe'le'grini-Issac, S. Lehe'ricy, J. Doyon and H. Benali, Partial correlation for functional brain interactivity investigation in functional MRI, NeuroImage 32 (2006), 228-237.

[18] B. Chai, D. Walther, D. Beck and F.-F. Li, Exploring functional connectivities of the human brain using multivariate information analysis, 23rd Annual Conference on Neural Information Processing Systems, Advances in Neural Information Processing Systems (NIPS) 22 (2009), 270-278.

[19] Z. Wang, W. Xu, F.A.S. Lucas and Y. Liu, Incorporating prior knowledge into Gene Network Study, Bioinformatics 29 (2013), 2633-2640.

[20] N. Zhong, J.M. Bradshaw, J. Liu and J.G. Taylor, Brain informatics, IEEE Intell. Syst. 26 (2011), 16-21. 